\title{
Change over from compound nuclear fission to quasi-fission
}

\author{
T. K.Ghosh ${ }^{1, a}$, K. Banerjee ${ }^{1}$, C. Bhattacharya ${ }^{1}$, S. Bhattacharya ${ }^{1}$, S. Kundu ${ }^{1}$, J.K. Meena ${ }^{1}$, G. Mukherjee ${ }^{1}$, S. \\ Mukhopadhyay $^{1}$, T. K. Rana ${ }^{1}$, K. S. Golda ${ }^{2}$, and P. Bhattacharya ${ }^{3}$ \\ 1 Variable Energy Cyclotron Centre, 1/AF Bidhan Nagar, Kolkata 700 064, India \\ 2 Inter University Accelerator Centre, New Delhi-110067, India \\ 3 Saha Institute of Nuclear Physics, 1/AF Bidhan Nagar, Kolkata 700 064, India
}

\begin{abstract}
Fission fragment mass distribution has been measured in two reactions to populate compound nucleus ${ }^{246} B k$. Both the target nuclei were deformed. However, entrance channel mass asymmetry of the two systems was on the either side of the Businaro Gallone mass asymmetry parameter. Near the Coulomb barrier, at similar excitation energies, the width of the fission fragment mass distribution was found to be significantly different for the ${ }^{14} N+{ }^{232} \mathrm{Th}$ reaction compared to the ${ }^{11} B+{ }^{235} U$ reaction. The entrance channel mass asymmetry was found to play a significant role in deciding the fusion process.
\end{abstract}

\section{Introduction}

In order to synthesise the heaviest elements fusion of two heavy nuclei is required. The fusion of two heavy nuclei to reach the island of stability in super heavy mass region has been studied extensively in recent years [1,2]. The direct evidence of the fusion leading to a super heavy nucleus is the production of the evaporation residue, while in most of the cases the super heavy nucleus decays by spontaneous fission. Hence the study of fusion-fission reactions becomes important to fix the initial conditions to maximize the probability for production as well as survival of the super heavy nucleus. Generally, the fusionfission process obeys statistical laws as the fission follows complete equilibration of the fused system of the colliding heavy ions in the entrance channel. Hence the fission reaction observables, such as the fragment mass and angular distributions, would follow statistical laws and become independent of the entrance channel variables, such as the mass asymmetry. However, in several systems, remarkable departures from the statistical theory predictions in fission fragment mass distributions have been observed with evidence of predominance of non-compound fission channels, particularly in near Coulomb barrier energies, with dependence on the entrance channel variables. It has been suggested [3] that in the multi-dimensional potential energy landscape, in the above barrier energies, the system preferentially moves over a fusion barrier to reach a composite system that reaches a meadow of evaporation residues. After emission of particles, the composite system undergoes binary fission following shape oscillations over a unconditional mass-symmetric fission barrier. However, as the excitation energy is lowered (i.e., at the near or below barrier energies), this path gets progressively blocked. But the

\footnotetext{
a e-mail: tilak@veccal.ernet.in
}

system, in the case of the highly deformed entrance channel nucleus (either target or projectile or both), reaches a mass-asymmetric fission barrier following a ridge along the fusion-hill and produces events with increased width of the mass distribution, which could be observed in experiments. Such observation has been reported for the deformed target of ${ }^{232} \mathrm{Th}$ in reaction with ${ }^{19} \mathrm{~F},{ }^{16} \mathrm{O}$, and ${ }^{12} \mathrm{C}$ [4]. The reaction mechanism as described above is termed as a nuclear orientation dependent quasi-fission reaction [5], in which the projectiles in contact with the tip region of the prolate target give rise to the quasi- fission events. The probability of such reaction increases for the mass flow preferentially from heavier mass to the lighter mass to form the composite system. Evidence of quasi-fission for the symmetric reaction populating low fissility composite system has been reported very recently [6]. Since the super heavy nuclei are of higher fissility and to be produced at lower excitation energy, thus study of fusion-fission dynamics of a higher fissility composite system near the Coulomb barrier (i.e., at low excitation energy with considerable fission cross section) is of prime interest. In this contribution, we report a sharp dependence of the entrance channel mass asymmetry in the fusion of two heavy ions near the Coulomb barrier to produced a highly fissile nucleus ${ }^{246} B k$.

\section{Experiment}

${ }^{246} B k$ nucleus was populated in the reactions ${ }^{11} B+{ }^{235} U$ and ${ }^{14} N+{ }^{232} T h$. The beam energies were judiciously chosen to populate the ${ }^{246} B k$ nucleus at similar excitation energies. Two large area multi-wire proportional counters were used to simultaneously detect the complimentary fission fragments [7]. The experiment was performed using pulsed beam of width about $1.1 \mathrm{~ns}$, with a pulse separation of 250

This is an Open Access article distributed under the terms of the Creative Commons Attribution-Noncommercial License, which permits unrestricted use, distribution, and reproduction in any noncommercial medium, provided the original work is properly cited. 


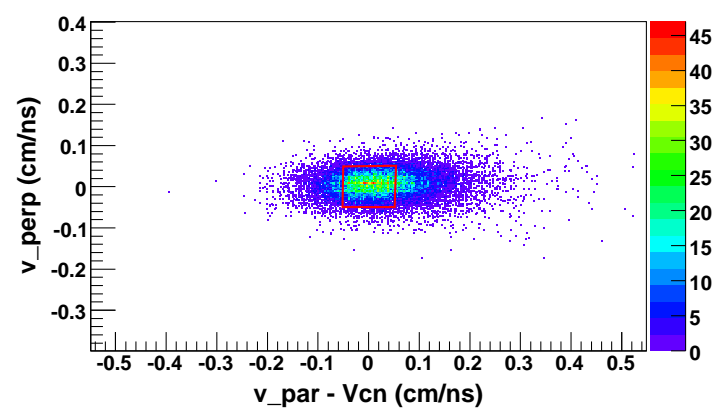

Fig. 1. Measured distribution of velocity of fissioning nuclei formed in the reaction ${ }^{14} N+{ }^{232} \mathrm{Th}$ at $E_{c} m=77.3 \mathrm{MeV}$. The (red) rectangle indicates the gate used to select the fusion fission events for mass determination.

ns, from the 15UD Pelletron at the Inter University Accelerator Centre (IUAC), New Delhi. Target of ${ }^{232} T h$ (on $200 \mu \mathrm{gm} / \mathrm{cm}^{2} \mathrm{Al}$ backing) and ${ }^{235} U$ (on $300 \mu \mathrm{gm} / \mathrm{cm}^{2} \mathrm{Ni}$ backing) of thickness $500 \mu \mathrm{gm} / \mathrm{cm}^{2}$ were used. The fission fragments were well separated from the elastic and quasielastic reaction channels, both from the event-time and energy loss spectra in the detectors. At low excitation energies close to the Coulomb barrier, the fission fragments are also produced in an incomplete fusion-fission reaction, in which only a part of the projectile fuses with the target and the incompletely fused binary system equilibrates and undergoes statistical fission reaction. Hence such a reaction mechanism, known as transfer fission (TF), needs to be separated experimentally from the compound nuclear and quasi fission reactions. The fission fragments from complete fusion events followed by fission were exclusively selected from the correlation of the velocities of the fissioning system $\left(V_{p a r}\right)$ in the beam direction relative to the recoil of the fused system and the velocity perpendicular to the reaction plane $\left(V_{\text {perp }}\right)$, as well as the correlation of the polar and azimuthal angles of the fragments $(\theta, \phi)$ with respect to the beam axis. The velocity distribution for ${ }^{14} N$ $+{ }^{232} \mathrm{Th}$ at $E_{c m}=77.3 \mathrm{MeV}$ are shown in Figure 1. The events due to the above two reaction channels (i.e., FF and TF) are also shown in a plot of the distribution of the polar folding angle in Figure 2. Figure 2 shows that the measured folding angle distribution of FF events is peaked around $165^{\circ}$ consistent with the expected value for the complete transfer of momentum of the projectile. The events due to incomplete fusion are peaked around a smaller folding angle. The masses were determined from the difference of the time-of-flights, polar and azimuthal angles, momentum and the recoil velocities for each event within the gate on $V_{p a r}-V_{C N}, V_{\text {perp }}$ as described [7,8].

It is to be noted that both the ${ }^{235} U$ and ${ }^{232} \mathrm{Th}$ targets chosen in our experiment to produce ${ }^{246} B k$ nucleus were deformed. However, the entrance channel mass asymmetries in two reactions were on the either side of the Businaro Gallone mass-asymmetry parameter value $\left(\alpha_{B G}=0.893\right)$ [9]. It is of interest to note that in the entrance channel, for

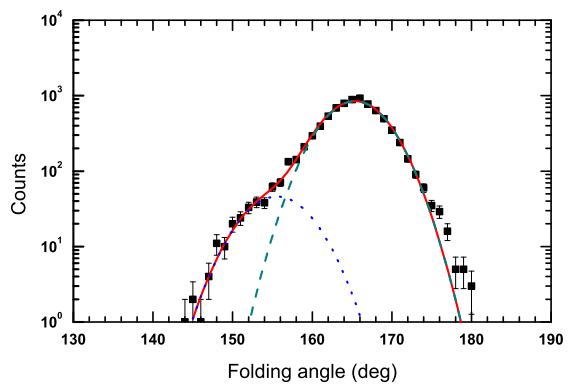

Fig. 2. Measured folding angle distributions of all fission fragments in the reaction ${ }^{14} N+{ }^{232} T h$ at $E_{c m}=77.3 \mathrm{MeV}$. Solid squares are the experimental points, (green) dashed and (blue) dotted lines represent the Gaussian fitting of the fusion fission (FF) and transfer induced fission (TF) events, respectively. The sum of the two Gaussian fittings is shown by the solid (red) line.

a small change in the entrance channel mass-asymmetry, the initial mass flow is from the lighter to heavier mass in the system ${ }^{11} B+{ }^{235} U(\alpha=0.911)$ while it is the reverse direction, i.e., from the heavier to lighter mass in the ${ }^{14} N+{ }^{232} T h(\alpha=0.886)$ system. Any inequality of the width of the mass distribution in the decay of the ${ }^{246} B k$ for the system with matched excitation and angular momentum in the entrance channel would be a confirmation of the effect of non compound fusion-fission paths in the multidimensional potential energy surfaces.

\section{Results}

The mass distribution plots, near and above the Coulomb barrier energies are shown in Figure 3 for ${ }^{11} B+{ }^{235} U$ and ${ }^{14} N+{ }^{232} T h$ systems. It can be observed that measured mass distributions are well fitted with single Gaussian distributions at all energies. The variation of standard deviation of the mass distributions with excitation energy for two reactions ${ }^{14} N+{ }^{232} T h$ and ${ }^{11} B+{ }^{235} U$ forming the same composite system ${ }^{246} B k$ is shown in Figure 4. Solid (red) line in the figure shows the calculated variation from the statistical theory [10] using the following relation $\sigma_{m}^{2}=$ $1 / \mathrm{k} .\left(E^{*} / a\right)^{1 / 2}$,where $E^{*}$ is the excitation energy at the scission point, $\mathrm{a}$ is the nuclear level density parameter. A value of the stiffness parameter $\mathrm{k}=0.0033 \mathrm{MeV} / u^{2}$ fitted the ${ }^{11} B+{ }^{235} U$ data well [11]. It is interesting to note that for the ${ }^{14} N+{ }^{232} T h$ reactions, not only there is a sudden increase of mass width $\left(\sigma_{m}\right)$ with decrease in excitation energy near the Coulomb barrier but also the magnitude is higher than that of the ${ }^{11} B+{ }^{235} U$ reactions over the entire range of excitation energies. We have observed that for the fusion of two heavy nuclei forming the compound nucleus ${ }^{246} B k$ through the reaction ${ }^{11} B+{ }^{235} U$ and ${ }^{14} \mathrm{~N}$ $+{ }^{232} \mathrm{Th}$ at the similar excitation energy, the fused system behaves differently and the reactions paths followed by the two systems, particularly around the Coulomb barrier, are significantly different. The target in both the cases are prolate deformed in the ground state while the projectiles are spherical; yet, the reaction paths followed by the 

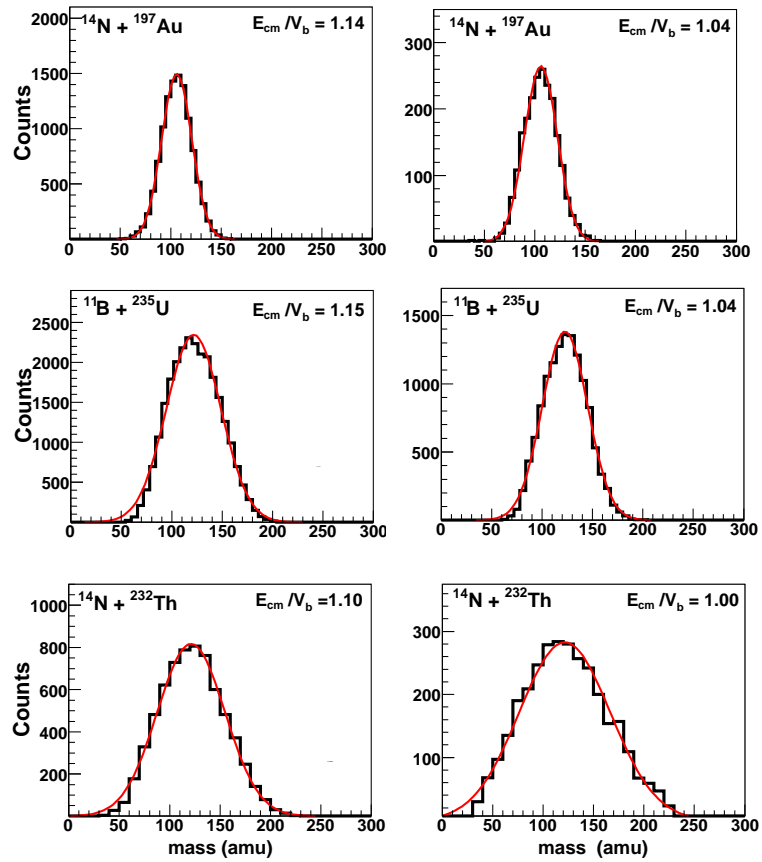

Fig. 3. Measured mass distributions for the reactions ${ }^{11} B+{ }^{235} U$ and ${ }^{14} N+{ }^{232} T h$ near the Coulomb barrier. The Gaussian fits are shown by the solid line.

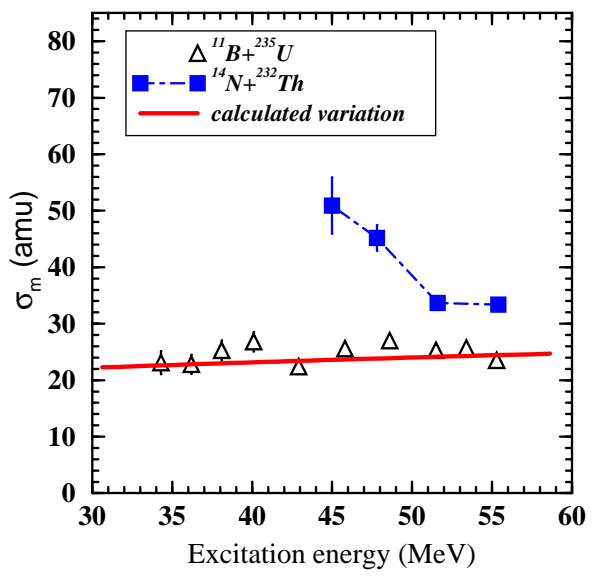

Fig. 4. Measured variation of $\sigma_{m}$ with excitation energy. Calculated variation is shown by solid (red) line. Position of Coulomb barrier is shown by the solid (blue) and dashed (black) arrows for ${ }^{14} N+{ }^{232} T h$ and ${ }^{11} B+{ }^{235} U$ reaction respectively.

two systems are quite different. For the ${ }^{14} N+{ }^{232} T h$ system, in the measured energy region, the reactions proceed mostly through the impact of the projectile on the polar region of the target nucleus and it drives the non-compact entrance channel shape to an almost mass symmetric saddle shape leading to increased mass width, rather than a compact equilibrated fused system, which undergoes fission through shape oscillations. The observed increase of the mass width with decrease in energy, in the case of ${ }^{14} \mathrm{~N}$ $+{ }^{232} T h$ system is due to quasi-fission phenomenon. How- ever, in case of the ${ }^{11} B+{ }^{235} U$ system, the initial separation in the entrance channel may not be sufficient to drive the system to a non-compact entrance channel to a mass asymmetric saddle shape. We also point out that, although the entrance channel mass asymmetries for the two systems do not differ much, in the two cases the flow of mass is completely different as the two nuclei fuse together. In the system ${ }^{14} N+{ }^{232} T h$, the entry point to the multidimensional potential surface is such that the system is driven to lower mass asymmetry leading to greater probability to diverge to a mass asymmetric saddle rather than a compact fused system, while the situation is just reverse in the case of ${ }^{11} B+{ }^{235} U$, where the system is driven to higher mass asymmetry and thus is more probable to reach a compact fused system and undergo statistical fission after equilibration.

\section{Conclusion}

In the fusion of ${ }^{246} \mathrm{Bk}$, the entrance channel mass asymmetry plays a crucial role in the reaction mechanism, particularly in the energy close to the Coulomb barrier. Even a small change in the entrance channel mass asymmetry, which effectively reverse the flow of mass in fusing the target and projectile, results quasi fission in reaction ${ }^{14} \mathrm{~N}+$ ${ }^{232} \mathrm{Th}$, while fusion fission for the reaction ${ }^{11} \mathrm{~B}+{ }^{235} U$. The entrance channel parameter was found to affect the fusion process sharply in the formation of the nucleus ${ }^{246} B k$.

\section{References}

1. Yu. Ts. Oganessian et al, Phys. Rev. C 75, (2006) 044602

2. Yu. Ts. Oganessian et al, Phys. Rev. C 76, (2007) 011601(R)

3. P. Moller and A. J. Sierk, Nature, Nature 422, (2003) 485

4. T. K. Ghosh et al, Phys. Lett. B 627, (2005) 26

5. D. J. Hinde et al, Phys. Rev. Lett. 74, (1995) 1295

6. R. Rafiei et al, Phys. Rev. C 77, (2008) 024606

7. T. K. Ghosh et al, Phys. Rev. C 79, (2009) 054607

8. T. K. Ghosh et al, Nucl. Instr. and Meth. A 540, (2005) 285

9. U. L. Businaro and S. Gallone, Nuovo Cimento 5, (1957) 315

10. B. B. Back et al, Phys. Rev. C 53, (1996) 1734

11. M. G. Itkis et al, Yad. Fiz 52, (1990) 23 